Vol. 46 (1992) [497-507]

\title{
CHARACTERISTIC SUBGROUPS OF RELATIVELY FREE GROUPS
}

\author{
ROger M. BRYANT
}

\begin{abstract}
A simple new proof is given of a result of Vaughan-Lee which implies that if $G$ is a relatively free nilpotent group of finite rank $k$ and nilpotency class $c$ with $c<k$ then the characteristic subgroups of $G$ are all fully invariant. It is proved that the condition $c<k$ can be weakened to $c<k+p-2$ when $G$ has p-power exponent for some prime $p$. On the other hand it is shown that for each prime $p$ there is a 2-generator relatively free $p$-group $G$ which is nilpotent of class $2 p$ such that the centre of $G$ is not fully invariant.
\end{abstract}

\section{INTRODUCTION}

For each positive integer $k$ let $F_{k}$ be the free group of rank $k$ freely generated by the set $\left\{x_{1}, \ldots, x_{k}\right\}$. The following result was proved in [7].

THEOREM 1. (Vaughan-Lee). If $C$ is a characteristic subgroup of $F_{k}$ which contains the $k$ th term $\gamma_{k}\left(F_{k}\right)$ of the lower central series of $F_{k}$ then $C$ is fully invariant.

One interesting consequence of this result is that every formation of finite nilpotent groups is subgroup closed - see [6]. A simple proof of Theorem 1 is given in Section 2 below.

A corollary of Theorem 1 is that if $G$ is a relatively free nilpotent group of finite rank $k$ and nilpotency class $c$ where $c<k$ then every characteristic subgroup of $G$ is fully invariant. (See [5] for basic facts about relatively free groups.) The following stronger result will be proved in Section 3 for the case where $G$ has prime-power exponent.

THEOREM 2. Let $p$ be a prime number and let $G$ be a relatively free nilpotent group of p-power exponent with finite rank $k$ and nilpotency class $c$, where $c<k+p-2$. Then every characteristic subgroup of $G$ is fully invariant.

In the case where $k=2$ and $p=2$ Theorem 2 is the best possible result of its sort because, as is well known, the relatively free group of rank 2 in the variety of groups of exponent 4 and class 2 has a characteristic subgroup which is not fully invariant (see, for example, [4, Section 9] and see also Section 6 below). I do not know how close Theorem 2 is to being best possible in general, but some information can be obtained

Received 4 December 1991

Copyright Clearance Centre, Inc. Serial-fee code: 0004-9729/92 \$A2.00+0.00. 
by module-theoretic methods. This is illustrated in Sections $4-6$. It is shown that for each odd prime $p$ there is a 2-generator relatively free p-group which is nilpotent of class $2 p-1$ and has a characteristic subgroup which is not fully invariant. The following result will also be proved.

Theorem 3. For each prime $p$ there is a 2 -generator relatively free $p$-group $G$ such that $G$ is nilpotent of class $2 p$ and the centre of $G$ is not fully invariant.

Similar examples of relatively free nilpotent groups of finite rank in which the centre is not fully invariant may possibly be known in the "folk-lore". A non-nilpotent example is certainly known; namely, the relatively free group of rank 2 in the variety generated by the non-abelian group of order 6 . In contrast, every relatively free group of infinite rank has fully invariant centre.

\section{Proof of Theorem 1}

If $F$ is a free group on a given free generating set $X$ and $x \in X$ we write $\delta_{x}$ for the "deletion" endomorphism of $F$ defined by $x \delta_{z}=1$ and $y \delta_{z}=y$ for all $y \in X \backslash\{x\}$. Furthermore, for all $w \in F, w\left(1-\delta_{x}\right)$ denotes $w\left(w \delta_{x}\right)^{-1}$.

As shown in [7], Theorem 1 is an easy consequence of the following lemma (Lemma 1). We shall not repeat the deduction of Theorem 1 here but give a proof of the lemma which avoids the complexity of the proof in [7].

LEMma 1. Let $C$ be a characteristic subgroup of $F_{k}$ which contains $\gamma_{k}\left(F_{k}\right)$ and let $w \in C$. Then $w \delta_{x_{i}} \in C(1 \leqslant i \leqslant k)$.

ProOf: The result is trivial if $k=1$, so we assume that $k \geqslant 2$. By symmetry, it is enough to prove that $w \delta_{x_{k}} \in C$. Write $w=w\left(x_{1}, \ldots, x_{k}\right)$. Let $F$ be the free group freely generated by $2 k$ elements $x_{1}, \ldots, x_{k}, y_{1}, \ldots, y_{k}$ and write $Y=\left\{y_{1}, \ldots, y_{k}\right\}$. Let $v$ be the element of $F$ defined by

and let

$$
\begin{gathered}
v=w\left(x_{2} x_{1} y_{1}^{-1}, x_{3} x_{2} y_{2}^{-1}, \ldots, x_{k} x_{k-1} y_{k-1}^{-1}, y_{1} y_{2} \ldots y_{k}\right) \\
v^{*}=v\left(1-\delta_{y_{1}}\right)\left(1-\delta_{y_{2}}\right) \ldots\left(1-\delta_{y_{k}}\right) .
\end{gathered}
$$

Thus $v^{*}$ is the product, in some order, of elements $v_{S}^{s(S)}$ where $S$ ranges over the subsets of $Y, \varepsilon(S)=(-1)^{|S|}$ and $v_{S}=v\left(\prod_{y \in S} \delta_{y}\right)$. Also, by $[5,33.38$ and 33.42], $\boldsymbol{v}^{*} \in \boldsymbol{\gamma}_{\boldsymbol{k}}(\boldsymbol{F})$.

Let $\xi: F \rightarrow F_{h}$ be the homomorphism defined by $x_{i} \xi=x_{i}$ and $y_{i} \xi=x_{i}$ for all $i$. Thus $v^{*} \xi \in \gamma_{k}\left(F_{k}\right)$ and so $v^{*} \xi \in C$. Also, $v^{*} \xi$ is the product of the elements $\left(v_{S} \xi\right)^{e(S)}$. We shall prove (i) $v_{Y} \xi=\left(w \delta_{x_{k}}\right) \alpha$ where $\alpha$ is an automorphism of $F_{k}$, and (ii) for all $S \neq Y, v_{S} \xi=w \beta$ where $\beta$ is an automorphism of $F_{k}$ (depending on $S$ ). It follows that $\left(v_{S} \xi\right)^{e(S)} \in C$ for all $S \neq Y$; whence $\left(w \delta_{z_{k}}\right) \alpha \in C$ and so $w \delta_{x_{k}} \in C$, as required. 
To prove (i) note that

$$
v_{Y} \xi=w\left(x_{2} x_{1}, x_{3} x_{2}, \ldots, x_{k} x_{k-1}, 1\right)=\left(w \delta_{x_{k}}\right) \alpha
$$

where $\alpha$ is the automorphism of $F_{k}$ defined by $x_{k} \alpha=x_{k}$ and $x_{i} \alpha=x_{i+1} x_{i}$ for $i<k$. To prove (ii), suppose $S \neq Y$ and, for $i=1, \ldots, k$, write $\mu_{i}=1$ if $y_{i} \in S$ and $\mu_{i}=0$ if $y_{i} \notin S$. Thus $v_{S} \xi=w \beta$ where $\beta$ is the endomorphism of $F_{k}$ defined by $x_{k} \beta=x_{1}^{1-\mu_{1}} \ldots x_{k}^{1-\mu_{k}}$ and $x_{i} \beta=x_{i+1} x_{i}^{\mu_{i}}$ for $i<k$. We shall show that $\beta$ is an automorphism of $F_{k}$. By the Hopf property of $F_{k}$ (see $[5,41.52]$ ) it is sufficient to show that $\beta$ is surjective. Thus it is sufficient to show that $x_{1}, \ldots, x_{k}$ all belong to the subgroup $\left\langle x_{1} \beta, \ldots, x_{k} \beta\right\rangle$.

Let $d$ be the smallest positive integer such that $y_{d} \notin S$. If $d<k$ then $x_{d} \beta=x_{d+1}$, $x_{d+1} \beta=x_{d+2} x_{d+1}^{\mu_{d+1}}, \ldots, x_{k-1} \beta=x_{k} x_{k-1}^{\mu_{k-1}}$, and so $x_{d+1}, \ldots, x_{k} \in\left\langle x_{1} \beta, \ldots, x_{k} \beta\right\rangle$. But $x_{k} \beta=x_{d} x_{d+1}^{1-\mu_{d+1}} \ldots x_{k}^{1-\mu_{k}}$. Hence $x_{d} \in\left\langle x_{1} \beta, \ldots, x_{k} \beta\right\rangle$. If $d>1$ then $x_{d-1} \beta=$ $x_{d} x_{d-1}, \ldots, x_{1} \beta=x_{2} x_{1}$, and so $x_{d-1}, \ldots, x_{1} \in\left\langle x_{1} \beta, \ldots, x_{k} \beta\right\rangle$.

\section{Proof of Theorem 2}

Let $G$ be as in the statement of the theorem and let $\left\{a_{1}, \ldots, a_{k}\right\}$ be a free generating set for $G$. The result is trivial if $k=1$ or $c=0$, so we assume that $k \geqslant 2$ and $c \geqslant 1$. Let $\Phi$ be the Frattini subgroup of $G$. Since $G$ is a finite $p$-group, $G / \Phi$ is an elementary abelian group of order $p^{k}$ and an endomorphism $\theta$ of $G$ is an automorphism if and only if $\left\{\left(a_{1} \theta\right) \Phi, \ldots,\left(a_{k} \theta\right) \Phi\right\}$ is a basis of $G / \Phi$.

Let $C$ be a characteristic subgroup of $G$. Let $u\left(x_{1}, \ldots, x_{k}\right)$ be an element of $F_{k}$ such that $u\left(a_{1}, \ldots, a_{k}\right) \in C$, and let $g_{1}, \ldots, g_{k} \in G$. It suffices to show that $u\left(g_{1}, \ldots, g_{k}\right) \in C$. Let $r$ be the rank of $\left\langle g_{1} \Phi, \ldots, g_{k} \Phi\right\rangle$. Then, for some subset $R$ of $\{1, \ldots, k\}$ of cardinality $r,\left\langle g_{1} \Phi, \ldots, g_{k} \Phi\right\rangle=\left\langle g_{i} \Phi: i \in R\right\rangle$. Suppose that $r \neq 0$, let $\sigma$ be a permutation of $\{1, \ldots, k\}$ such that $\{1, \ldots, r\}=R \sigma$, and let $\tilde{u}\left(x_{1}, \ldots, x_{k}\right)=u\left(x_{1 \sigma}, \ldots, x_{k \sigma}\right)$. Then

$$
\tilde{u}\left(a_{1}, \ldots, a_{k}\right)=u\left(a_{1 \sigma}, \ldots, a_{k \sigma}\right)=u\left(a_{1}, \ldots, a_{k}\right) \sigma^{*},
$$

where $\sigma^{*}$ is an automorphism of $G$. Thus $\tilde{u}\left(a_{1}, \ldots, a_{k}\right) \in C$. Also, $u\left(g_{1}, \ldots, g_{k}\right)=$ $\tilde{u}\left(g_{1 \sigma^{-1}}, \ldots, g_{k \sigma^{-1}}\right)$ and, by choice of $\sigma,\left\langle g_{1 \sigma^{-1}} \Phi, \ldots, g_{r \sigma^{-1}} \Phi\right\rangle=\left\langle g_{1} \Phi, \ldots, g_{k} \Phi\right\rangle$. Thus (by considering $\tilde{u}$ instead of $u$ ) we may reduce to the case where $R=\{1, \ldots, r\}$.

Hence there are elements $t_{r+1}\left(x_{1}, \ldots, x_{r}\right), \ldots, t_{k}\left(x_{1}, \ldots, x_{r}\right)$ of $F_{r}$ and elements $f_{r+1}, \ldots, f_{k}$ of $\Phi$ such that $g_{i}=t_{i}\left(g_{1}, \ldots, g_{r}\right) f_{i}(r+1 \leqslant i \leqslant k)$. Since $\left\{g_{1} \Phi, \ldots, g_{r} \Phi\right\}$ is contained in a basis of $G / \Phi$, there is an automorphism $\theta$ of $G$ such that $a_{i} \theta=g_{i}(1 \leqslant i \leqslant r)$. It is enough to prove that $u\left(g_{1}, \ldots, g_{k}\right) \theta^{-1} \in C$. But

$$
u\left(g_{1}, \ldots, g_{k}\right) \theta^{-1}=u\left(a_{1}, \ldots, a_{r}, t_{r+1}\left(a_{1}, \ldots, a_{r}\right) h_{r+1}, \ldots, t_{k}\left(a_{1}, \ldots, a_{r}\right) h_{k}\right)
$$


where $h_{i}=f_{i} \theta^{-1} \in \Phi(r+1 \leqslant i \leqslant k)$. Let

$$
w\left(x_{1}, \ldots, x_{k}\right)=u\left(x_{1}, \ldots, x_{r}, t_{r+1}\left(x_{1}, \ldots, x_{r}\right) x_{r+1}, \ldots, t_{k}\left(x_{1}, \ldots, x_{r}\right) x_{k}\right) .
$$

Thus we wish to prove that $w\left(a_{1}, \ldots, a_{r}, h_{r+1}, \ldots, h_{k}\right) \in C$. Note that $w\left(a_{1}, \ldots, a_{k}\right)$ $=u\left(a_{1}, \ldots, a_{k}\right) \tau$ where $\tau$ is an automorphism of $G$. Thus $w\left(a_{1}, \ldots, a_{k}\right) \in C$ and it is enough to prove the following lemma, which also covers the case where $r=0$. The proof is a modification of the proof of Lemma 1 .

Lemma 2. With $G$ as in the statement of Theorem 2 , let $\left\{a_{1}, \ldots, a_{k}\right\}$ be a free generating set for $G$, let $C$ be a characteristic subgroup of $G$, and let $w\left(x_{1}, \ldots, x_{k}\right)$ be an element of $F_{k}$ such that $w\left(a_{1}, \ldots, a_{k}\right) \in C$. Then, for all $r \in\{0,1, \ldots, k\}$ and all $h_{r+1}, \ldots, h_{k} \in \Phi$ (where $\Phi$ is the Frattini subgroup of $G), w\left(a_{1}, \ldots, a_{r}, h_{r+1}, \ldots, h_{k}\right)$ belongs to $C$.

Proof: As before we may assume that $k \geqslant 2$ and $c \geqslant 1$. The result is trivially true if $r=k$. Thus, using downward induction on $r$, we may assume that $r<k$ and the result is true when $r$ is replaced by $r+1$. Let $F$ be the free group freely generated by a set $\left\{x_{1}, \ldots, x_{k}\right\} \cup Y$ where $Y$ consists of the $k+p-2$ elements $y_{1}, \ldots, y_{r}, y_{r+1}^{(1)}, \ldots, y_{r+1}^{(p-1)}, y_{r+2}, \ldots, y_{k}$. Let $\xi: F \rightarrow G$ be the homomorphism defined by $x_{i} \xi=a_{i}$ for all $i, y_{i} \xi=a_{i}$ for all $i \neq r+1$, and $y_{r+1}^{(j)} \xi=a_{r+1}$ for all $j$. Let $\alpha$ be the automorphism of $G$ defined by $a_{i} \alpha=a_{i+1} a_{i}(1 \leqslant i \leqslant r)$ and $a_{i} \alpha=a_{i}$ $(r+1 \leqslant i \leqslant k)$, and write $h_{i}^{\prime}=h_{i} \alpha(r+1 \leqslant i \leqslant k)$. Choose elements $u_{r+1}, \ldots, u_{k}$ of $\left\langle x_{1}, \ldots, x_{k}\right\rangle$ such that $u_{i} \xi=h_{i}^{\prime}(r+1 \leqslant i \leqslant k)$. Let $v$ be the element of $F$ defined by

$$
v=w\left(x_{2} x_{1} y_{1}^{-1}, \ldots, x_{r+1} x_{r} y_{r}^{-1}, y_{1} \ldots y_{r} y_{r+1}^{(1)} \ldots y_{r+1}^{(p-1)} y_{r+2} \ldots y_{k} u_{r+1}, u_{r+2}, \ldots, u_{k}\right)
$$

and let $v^{*}=v\left(\prod_{y \in Y}\left(1-\delta_{y}\right)\right)$ where the elements of $Y$ are taken in some arbitrary order. Thus, by $\left[5,33.38\right.$ and 33.42], $v^{*} \in \gamma_{k+p-2}(F)$, and so $v^{*} \xi \in \gamma_{k+p-2}(G)=\{1\}$. In particular, $v^{*} \xi \in C$. For each subset $S$ of $Y$ let $v_{S}=v\left(\prod_{y \in S} \delta_{y}\right)$. As in the proof of Lemma 1, it suffices to prove (i) $v_{Y} \xi=w\left(a_{1}, \ldots, a_{r}, h_{r+1}, \ldots, h_{k}\right) \alpha$, and (ii) for all $S \neq Y, v_{s} \xi \in C$.

To prove (i) note that

$$
v_{Y} \xi=w\left(a_{2} a_{1}, \ldots, a_{r+1} a_{r}, h_{r+1}^{\prime}, \ldots, h_{k}^{\prime}\right)=w\left(a_{1}, \ldots, a_{r}, h_{r+1}, \ldots, h_{h}\right) \alpha,
$$

as required. To prove (ii), suppose $S \neq Y$, write

$$
\nu=\left|\left\{y_{r+1}^{(1)}, \ldots, y_{r+1}^{(p-1)}\right\} \cap S\right|
$$


and, for $i \in\{1, \ldots, r, r+2, \ldots, k\}$, write $\mu_{i}=1$ if $y_{i} \in S$ and $\mu_{i}=0$ if $y_{i} \notin S$. Thus $v_{S} \xi=w\left(b_{1}, \ldots, b_{k}\right)$ where $b_{1}=a_{2} a_{1}^{\mu_{1}}, \ldots, b_{\tau}=a_{r+1} a_{r}^{\mu_{r}}$,

$$
b_{r+1}=a_{1}^{1-\mu_{1}} \ldots a_{r}^{1-\mu_{r}} a_{r+1}^{p-1-\nu} a_{r+2}^{1-\mu_{r+2}} \ldots a_{h}^{1-\mu_{k}} h_{r+1}^{\prime} \text {, }
$$

$b_{r+2}=h_{r+2}^{\prime}, \ldots, b_{k}=h_{k}^{\prime}$. It is enough to prove that $\left\langle b_{1} \Phi, \ldots, b_{r+1} \Phi\right\rangle$ has rank $r+1$, for then there is an automorphism $\beta$ of $G$ such that $a_{i} \beta=b_{i}(1 \leqslant i \leqslant r+1)$ which gives

$$
v_{S} \xi=w\left(a_{1}, \ldots, a_{r+1}, h_{r+2}^{\prime} \beta^{-1}, \ldots, h_{k}^{\prime} \beta^{-1}\right) \beta
$$

and so $v_{S} \xi \in C$ by the inductive hypothesis.

Suppose first that $\left\{y_{1}, \ldots, y_{r}\right\} \subseteq S$. Then

$$
\left\langle b_{1}, \ldots, b_{r+1}\right\rangle=\left\langle a_{2} a_{1}, \ldots, a_{r+1} a_{r}, a_{r+1}^{p-1-\nu} a_{r+2}^{1-\mu_{r+2}} \ldots a_{k}^{1-\mu_{k}} h_{r+1}^{\prime}\right\rangle
$$

and either $\mu_{i}=0$ for some $i \in\{r+2, \ldots, k\}$ or $0 \leqslant \nu<p-1$. It follows easily that $\left\langle b_{1} \Phi, \ldots, b_{r+1} \Phi\right\rangle$ has rank $r+1$.

Suppose finally that $\left\{y_{1}, \ldots, y_{r}\right\}$ is not contained in $S$ and let $N=\left\langle a_{r+2}, \ldots, a_{k}\right\rangle \Phi$. It suffices to show that $\left\langle b_{1} N, \ldots, b_{r+1} N\right\rangle$ has rank $r+1$. But

$$
\left\langle b_{1} N, \ldots, b_{r+1} N\right\rangle=\left\langle a_{2} a_{1}^{\mu_{1}} N, \ldots, a_{r+1} a_{r}^{\mu_{r}} N, a_{1}^{1-\mu_{1}} \ldots a_{r}^{1-\mu_{r}} a_{r+1}^{p-1-\nu} N\right\rangle
$$

where $\mu_{i}=0$ for some $i \in\{1, \ldots, r\}$. By the method of Lemma 1 we can show that $a_{1} N, \ldots, a_{r+1} N$ all belong to $\left\langle b_{1} N, \ldots, b_{r+1} N\right\rangle$, and this gives the required result.

\section{ENDOMORPHISMS AND MODULES}

In the remainder of the paper we describe the construction of some relatively free p-groups with characteristic subgroups which are not fully invariant.

Let $p$ be a prime number and $k$ a positive integer, $k \geqslant 2$. Define subgroups $\lambda_{c}\left(F_{k}\right)$ of $F_{k}$ for each positive integer $c$ by $\lambda_{1}\left(F_{k}\right)=F_{k}$ and

$$
\lambda_{c+1}\left(F_{k}\right)=\lambda_{c}\left(F_{k}\right)^{p}\left[\lambda_{c}\left(F_{k}\right), F_{k}\right]
$$

(see [2, VIII.1.4 and VIII.1.5]). Thus each $\lambda_{c}\left(F_{k}\right)$ is a fully invariant subgroup of $F_{k}$, $\lambda_{c+1}\left(F_{k}\right) \subseteq \lambda_{c}\left(F_{k}\right)$ and $\lambda_{c}\left(F_{k}\right) / \lambda_{c+1}\left(F_{k}\right)$ is a finite elementary abelian $p$-group. We write $U_{c}=\lambda_{c}\left(F_{k}\right) / \lambda_{c+1}\left(F_{k}\right)$ and regard $U_{c}$ as a vector space over the field $G F(p)$ of $p$ elements. Furthermore, we write $U=U_{1}$. Thus $U$ has dimension $k$.

For each $c$ let $\operatorname{End}\left(U_{c}\right)$ be the set of all linear transformations of $U_{c}$. It is usual to regard End $\left(U_{c}\right)$ as a ring, but for our purposes here the additive structure of End $\left(U_{c}\right)$ is irrelevant and we simply regard $\operatorname{End}\left(U_{c}\right)$ as a monoid under the operation of composition of functions. As usual we write $\mathrm{GL}\left(U_{c}\right)$ for the group of invertible elements 
of End $\left(U_{c}\right)$. Every endomorphism of $F_{k}$ induces on $U_{c}$ an element of End $\left(U_{c}\right)$. Since $F_{k}$ is free, each element $\zeta$ of End $(U)$ is induced by some endomorphism of $F_{k}$. This endomorphism induces an element of End $\left(U_{c}\right)$ which, by [2, VIII.1.7a], depends only upon $\zeta$ and not on the choice of endomorphism of $F_{k}$. Thus we obtain a monoid homomorphism End $(U) \rightarrow$ End $\left(U_{c}\right)$ and, by restriction, a group homomorphism $\mathrm{GL}(U) \longrightarrow \mathrm{GL}\left(U_{c}\right)$. Hence $U_{c}$ may be regarded as a module for $\operatorname{End}(U)$ and $\mathrm{GL}(U)$.

Note that $F_{k} / \lambda_{c+1}\left(F_{k}\right)$ is a finite relatively free p-group of rank $k$ and nilpotency class at most $c$. (We shall see below that it has class exactly $c$.) Furthermore, $U_{c}$ is a central subgroup of $F_{k} / \lambda_{c+1}\left(F_{k}\right)$. Suppose that $V$ is a subgroup of $U_{c}$. Then it is straightforward to verify that $V$ is fully invariant as a subgroup of $F_{k} / \lambda_{c+1}\left(F_{k}\right)$ if and only if it is an End $(U)$-submodule of $U_{c}$ and $V$ is characteristic in $F_{k} / \lambda_{c+1}\left(F_{k}\right)$ if and only if it is a GL $(U)$-submodule of $U_{c}$. Thus we shall investigate the submodule structure of $U_{c}$.

Let $A$ be the free associative algebra (without identity element) over GF (p) on the free generating set $\left\{x_{1}, \ldots, x_{k}\right\}$. Thus $A=A_{1} \oplus A_{2} \oplus \ldots$ where, for each $c, A_{c}$ is the subspace of $A$ spanned by all monomials of degree $c$ in $x_{1}, \ldots, x_{k}$. We identify $U$ with $A_{1}$ in the obvious way. Thus $A_{1}$ is an End $(U)$-module. Since $A$ is free, the action of $\operatorname{End}(U)$ on $A_{1}$ can be extended to $A$ so that each element of $\operatorname{End}(U)$ acts as an algebra endomorphism of $A$. Under this action $A$ is an $\operatorname{End}(U)$-module and each $A_{c}$ is a submodule.

The associative algebra $A$ also carries the structure of a Lie algebra over GF $(p)$, the Lie multiplication being the "commutator" operation defined by $[v, w]=v w-w v$ for all $v, w \in A$. Let $L$ be the Lie subalgebra generated by $\left\{x_{1}, \ldots, x_{k}\right\}$. Then, as is well known, $L$ is a free Lie algebra on $\left\{x_{1}, \ldots, x_{k}\right\}$ (see [3, Theorem 5.9]). Also $L=L_{1} \oplus L_{2} \oplus \ldots$ where $L_{c}=L \cap A_{c}$ for all $c$, and, in particular, $L_{1}=A_{1}=U$. It is easy to verify that $L$ and the $L_{c}$ are $\operatorname{End}(U)$-submodules of $A$.

The submodule structure of $U_{c}$ is closely related to that of $L$. In explaining the connection we shall follow the presentation in [1]: see [1] for references to original sources.

For each positive integer $c$ there is a certain group homomorphism $\phi_{c}: \lambda_{c}\left(F_{k}\right) \rightarrow$ $A$ (with $A$ regarded as a group under addition). In the case where $p$ is odd these homomorphisms are determined by the following properties:

and

$$
x_{i} \phi_{1}=x_{i}(1 \leqslant i \leqslant k), \quad f^{p} \phi_{c+1}=f \phi_{c},
$$

for all $c \geqslant 1, f \in \lambda_{c}\left(F_{k}\right)$ and $g \in \lambda_{1}\left(F_{k}\right)=F_{k}$. For $p=2$ the only difference is in the formula for $f^{p} \phi_{c+1}$ with $c=1$ : this becomes

$$
f^{2} \phi_{2}=f \phi_{1}+\left(f \phi_{1}\right)^{2}
$$


for all $f \in \lambda_{1}\left(F_{k}\right)$.

The kernel of $\phi_{c}$ is equal to $\lambda_{c+1}\left(F_{k}\right)$ for all $p, c$ (see [1]). Thus $\phi_{c}$ induces a vector space monomorphism $\tilde{\phi}_{c}: U_{c} \rightarrow A$. As noted in [1], $\tilde{\phi}_{c}$ is a GL $(U)$-module monomorphism, and a similar proof shows that $\tilde{\phi}_{c}$ is in fact an End $(U)$-module monomorphism. For $p \neq 2$ the image of $\tilde{\phi}_{c}$ is easily calculated to be $L_{1}+\ldots+L_{c}$ : thus $U_{c}$ is isomorphic to $L_{1}+\ldots+L_{c}$ as $\operatorname{End}(U)$-module. In the case $p=2$, let $E$ be the subspace of $A_{1}+A_{2}$ spanned by the elements $x_{i}+x_{i}^{2}(1 \leqslant i \leqslant k)$ and $\left[x_{i}, x_{j}\right](1 \leqslant i<j \leqslant k)$. Then the image of $\tilde{\phi}_{1}$ is $L_{1}$, the image of $\tilde{\phi}_{2}$ is $E$, and, for $c \geqslant 3$, the image of $\tilde{\phi}_{c}$ is $E+L_{3}+\ldots+L_{c}$. Thus $U_{c}$ is again determined up to isomorphism.

Clearly $\gamma_{c}\left(F_{k}\right) \subseteq \lambda_{c}\left(F_{k}\right)$ for all $c$. It is easy to prove by induction on $c$ that $\gamma_{c}\left(F_{k}\right) \phi_{c}=L_{c}$ for all $c$. Hence $\gamma_{c}\left(F_{k}\right)$ is not contained in $\lambda_{c+1}\left(F_{k}\right)$ and the group $F_{k} / \lambda_{c+1}\left(F_{k}\right)$ has class exactly $c$.

In the remainder of this paper we take $k=2$ and write $z$ as an abbreviation for the element $\left[x_{1}, x_{2}\right]$ of $L$. Thus $z$ spans $L_{2}$. Note that, for all $\zeta \in \operatorname{End}(U), z \zeta=\operatorname{det}(\zeta) z$. Commutators in $L$ will be written with a left-normed convention. Also, we shall need to use the well known fact that if $v$ and $w$ are elements of $L$ such that $[v, w]=0$ and $w \neq 0$ then $v$ is a scalar multiple of $w$. This follows, for example, from [3, Theorem 5.10]. We shall now consider separately the cases where $p$ is odd (Section 5) and $p=2$ (Section 6).

\section{ODD CHARACTERISTIC}

Suppose that $k=2$ and $p$ is odd. We shall first show that $L_{1}+\ldots+L_{2 p-1}$ has a GL(U)-submodule which is not an $\operatorname{End}(U)$-submodule. Let $s_{1}$ and $s_{2}$ be the elements of $L_{2 p-1}$ defined by $s_{1}=\left[x_{1}, z, \ldots, z\right]$ and $s_{2}=\left[x_{2}, z, \ldots, z\right]$. (Here and subsequently $z, \ldots, z$ will denote a sequence of $p-1$ copies of $z$.) Let $V$ be the subspace of $L_{1}+L_{2 p-1}$ spanned by $x_{1}+s_{1}$ and $x_{2}+s_{2}$; that is, $V=\left\langle x_{1}+s_{1}, x_{2}+s_{2}\right\rangle$. Note that, for $\zeta \in \mathrm{GL}(U)$ and $i=1,2$,

$$
s_{i} \zeta=(\operatorname{det}(\zeta))^{p-1}\left[x_{i} \zeta, z, \ldots, z\right]=\left[x_{i} \zeta, z, \ldots, z\right] .
$$

It follows that $V$ is a $G L(U)$-submodule of $L_{1}+\ldots+L_{2 p-1}$ isomorphic to $L_{1}$. But $V$ is not an $\operatorname{End}(U)$-submodule because if $\eta$ is the element of $\operatorname{End}(U)$ which satisfies $x_{1} \eta=x_{1}$ and $x_{2} \eta=0$ on $A_{1}$ then $\left(x_{1}+s_{1}\right) \eta=x_{1} \notin V$. Thus $U_{2 p-1}$ has a $\mathrm{GL}(U)$-submodule which is not an $\operatorname{End}(U)$-submodule, and so the relatively free group $F_{2} / \lambda_{2 p}\left(F_{2}\right)$ has a characteristic subgroup which is not fully invariant. As proved in Section $4, F_{2} / \lambda_{2 p}\left(F_{2}\right)$ has class $2 p-1$.

We now move towards the proof of Theorem 3 (in the case where $p$ is odd). Let 
$t_{1}, t_{2}, t_{3}, t_{4}$ be the elements of $L_{2 p}$ defined by

$$
\begin{array}{ll}
t_{1}=\left[x_{1}, z, \ldots, z, x_{2}\right]=\left[s_{1}, x_{2}\right], & t_{2}=\left[x_{2}, z, \ldots, z, x_{1}\right]=\left[s_{2}, x_{1}\right], \\
t_{3}=\left[x_{1}, z, \ldots, z, x_{1}\right]=\left[s_{1}, x_{1}\right], & t_{4}=\left[x_{2}, z, \ldots, z, x_{2}\right]=\left[s_{2}, x_{2}\right] .
\end{array}
$$

LEMMA 3. The elements $t_{1}, t_{2}, t_{3}$ and $t_{4}$ are linearly independent,

$$
\begin{aligned}
&\left\langle t_{1}, t_{2}, t_{3}, t_{4}\right\rangle \cap\left[L_{2 p-1}, x_{1}\right]=\left\langle t_{2}, t_{3}\right\rangle \\
& \text { and } \quad\left\langle t_{1}, t_{2}, t_{3}, t_{4}\right\rangle \cap\left[L_{2 p-1}, x_{2}\right]=\left\langle t_{1}, t_{4}\right\rangle .
\end{aligned}
$$

Proof: We work inside $A$ and use the fact that the monomials of $A$ form a basis of $A$. Let $\theta: A \rightarrow A$ be the linear transformation which fixes $\left(x_{2} x_{1}\right)^{p-2} x_{1}^{2} x_{2}^{2}$ and $x_{2}^{3} x_{1}\left(x_{1} x_{2}\right)^{p-2}$ but maps all other monomials to 0 . Then it is straightforward to verify that

and

$$
t_{1} \theta=(p-1)\left(x_{2} x_{1}\right)^{p-2} x_{1}^{2} x_{2}^{2}
$$

$$
t_{4} \theta=x_{2}^{3} x_{1}\left(x_{1} x_{2}\right)^{p-2} \text {. }
$$

But clearly $v \theta=0$ for all $v \in\left[L_{2 p-1}, x_{1}\right]$. It follows that $t_{1}$ and $t_{4}$ are linearly independent and

$$
\left\langle t_{1}, t_{4}\right\rangle \cap\left[L_{2 p-1}, x_{1}\right]=\{0\} .
$$

Similarly, $t_{2}$ and $t_{3}$ are linearly independent and

$$
\left\langle t_{2}, t_{3}\right\rangle \cap\left[L_{2 p-1}, x_{2}\right]=\{0\} .
$$

The result follows.

Let $W=\left\langle z+t_{1},-z+t_{2}, t_{3}, t_{4}\right\rangle$. Then it is easy to verify that $W$ is an $\operatorname{End}(U)$ submodule of $L_{2}+L_{2 p}$. Furthermore, the following result holds.

Lемма 4. $V=\left\{v \in L:\left[v, x_{i}\right] \in W\right.$ for $\left.i=1,2\right\}$.

Proof: Clearly $\left[v, x_{1}\right] \in W$ and $\left[v, x_{2}\right] \in W$ for all $v \in V$. Conversely, let $v$ be an element of $L$ such that $\left[v, x_{1}\right] \in W$ and $\left[v, x_{2}\right] \in W$. We shall prove that $v \in V$.

Write $v=v_{1}+v_{2}+\ldots$ where $v_{j} \in L_{j}$ for all $j$. Then, for $j \notin\{1,2 p-1\}$, $\left[v_{j}, x_{1}\right]=0$ and $\left[v_{j}, x_{2}\right]=0$. Hence $v_{j}=0$ for all $j \notin\{1,2 p-1\}$. Thus $v \in$ $L_{1}+L_{2 p-1}$.

Let $\left\{s_{1}, \ldots, s_{n}\right\}$ be any basis for $L_{2 p-1}$ where $s_{1}=\left[x_{1}, z, \ldots, z\right]$ and $s_{2}=\left[x_{2}, z, \ldots, z\right]$ as before. If $w \in L_{2 p-1}$ and $\left[w, x_{1}\right]=0$ then $w=0$. Thus $\left[s_{1}, x_{1}\right], \ldots,\left[s_{n}, x_{1}\right]$ are linearly independent elements of $L_{2 p}$. Since $v \in L_{1}+L_{2 p-1}$ we can write

$$
v=\mu_{1} x_{1}+\mu_{2} x_{2}+\nu_{1} s_{1}+\ldots+\nu_{n} s_{n}
$$


where $\mu_{1}, \mu_{2}, \nu_{1}, \ldots, \nu_{n} \in \mathrm{GF}(p)$. Thus

$$
\left[v, x_{1}\right]=-\mu_{2} z+\nu_{1}\left[s_{1}, x_{1}\right]+\ldots+\nu_{n}\left[s_{n}, x_{1}\right] \in W .
$$

It follows that

$$
\nu_{1}\left[s_{1}, x_{1}\right]+\ldots+\nu_{n}\left[s_{n}, x_{1}\right] \in\left(t_{1}, t_{2}, t_{3}, t_{4}\right) \cap\left[L_{2 p-1}, x_{1}\right] .
$$

But, by Lemma 3,

$$
\left\langle t_{1}, t_{2}, t_{3}, t_{4}\right\rangle \cap\left[L_{2 p-1}, x_{1}\right]=\left\langle\left[s_{1}, x_{1}\right],\left[s_{2}, x_{1}\right]\right\rangle .
$$

Thus $\nu_{j}=0$ for $j>2$ and we can write

$$
v=\mu_{1} x_{1}+\mu_{2} x_{2}+\nu_{1} s_{1}+\nu_{2} s_{2}
$$

Hence

$$
\left[v, x_{1}\right]=-\mu_{2} z+\nu_{1} t_{3}+\nu_{2} t_{2}=\left(\nu_{2}-\mu_{2}\right) z+\nu_{1} t_{3}+\nu_{2}\left(-z+t_{2}\right)
$$

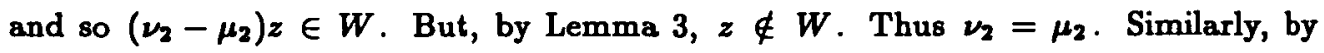
consideration of $\left[v, x_{2}\right], \nu_{1}=\mu_{1}$. Thus

$$
v=\mu_{1}\left(x_{1}+s_{1}\right)+\mu_{2}\left(x_{2}+s_{2}\right) \in V,
$$

as required.

We shall now make use of the properties of the maps $\phi_{c}$ given in Section 4. Let $M$ be the inverse image of $V$ under $\phi_{2 p-1}$ and let $N$ be the inverse image of $W$ under $\phi_{2 p}$. Thus

$$
\lambda_{2 p+1}\left(F_{2}\right) \subseteq N \subseteq \lambda_{2 p}\left(F_{2}\right) \subseteq M \subseteq \lambda_{2 p-1}\left(F_{2}\right) .
$$

Since $W$ is an End $(U)$-submodule of $L_{1}+\ldots+L_{2 p}, N / \lambda_{2 p+1}\left(F_{2}\right)$ is a fully invariant subgroup of $F_{2} / \lambda_{2 p+1}\left(F_{2}\right)$, and so $N$ is a fully invariant subgroup of $F_{2}$. Let $G=$ $F_{2} / N$. Then $G$ is a 2-generator relatively free p-group which is nilpotent of class at most $2 p$. But

$$
\left(N \gamma_{2 p}\left(F_{2}\right)\right) \phi_{2 p}=W+L_{2 p} \neq W=N \phi_{2 p} .
$$

Thus $N_{\gamma_{2 p}}\left(F_{2}\right) \neq N$ and $G$ has class exactly $2 p$.

Since $V$ is not an $\operatorname{End}(U)$-submodule of $L_{1}+\ldots+L_{2 p-1}, M / \lambda_{2 p}\left(F_{2}\right)$ is not a fully invariant subgroup of $F_{2} / \lambda_{2 p}\left(F_{2}\right)$. Thus $M / N$ is not a fully invariant subgroup of $G$. To complete the proof of Theorem 3 (in the case where $p$ is odd) we shall prove that $Z(G)=M / N$.

Let $f \in M$. Then $f \phi_{2 p-1} \in V$ and so, by Lemma $4,\left[f \phi_{2 p-1}, x_{i}\right] \in W$ for $i=1,2$. Thus $\left[f, x_{i}\right] \phi_{2 p} \in W$ and so $\left[f, x_{i}\right] \in N$ for $i=1,2$. Thus $M / N \subseteq Z(G)$.

Conversely, suppose that $f \in F_{2}$ and $\left[f, x_{i}\right] \in N$ for $i=1,2$. We first prove by induction on $c$ that $f \in \lambda_{c}\left(F_{2}\right)$ for $c=1, \ldots, 2 p-1$. Clearly $f \in \lambda_{1}\left(F_{2}\right)$. Suppose 
$f \in \lambda_{c}\left(F_{2}\right)$ where $c<2 p-1$. Then, since $N \subseteq \lambda_{c+2}\left(F_{2}\right),\left[f, x_{i}\right] \phi_{c+1}=0$ for $i=1,2$, and so $\left[f \phi_{c}, x_{i}\right]=0$ for $i=1,2$. Thus $f \phi_{c}=0$ and so $f \in \lambda_{c+1}\left(F_{2}\right)$. Therefore $f \in \lambda_{2 p-1}\left(F_{2}\right)$ and we can apply the map $\phi_{2 p-1}$. Thus, for $i=1,2$,

$$
\left[f \phi_{2 p-1}, x_{i}\right]=\left[f, x_{i}\right] \phi_{2 p} \in W .
$$

By Lemma 4 it follows that $f \phi_{2 p-1} \in V$. Thus $f \in M$. Consequently $Z(G) \subseteq M / N$ and so $Z(G)=M / N$ as required.

\section{Characteristic 2}

Suppose that $k=2$ and $p=2$. Let $E$ be as defined in Section 4: thus $E$ is the image of $\tilde{\phi}_{2}$. It is easily verified that the subspace of $E$ spanned by the elements $x_{1}+x_{1}^{2}+z$ and $x_{2}+x_{2}^{2}+z$ is a GL $(U)$-submodule but not an End $(U)$-submodule. Thus $F_{2} / \lambda_{3}\left(F_{2}\right)$ has a characteristic subgroup which is not fully invariant - this is the example referred to in Section 1. But it does not seem possible to use this example to create a 2-generator relatively free 2-group of class 3 whose centre is not fully invariant. It seems necessary to go to a group of class 4 . Thus we prove Theorem 3 in the case $p=2$. The proof is similar to that for $p$ odd, but rather easier. We omit some of the details.

Let $V_{(2)}$ be the subspace of $E+L_{3}$ spanned by the elements $x_{1}+x_{1}^{2}+z+\left[z, x_{1}\right]$ and $x_{2}+x_{2}^{2}+z+\left[z, x_{2}\right]$. It is easily verified that $V_{(2)}$ is a $\mathrm{GL}(U)$-submodule but not an $\operatorname{End}(U)$-submodule.

Let $W_{(2)}$ be the subspace of $E+L_{3}+L_{4}$ spanned by

$$
\left[z, x_{1}\right]+\left[z, x_{1}, x_{1}\right], \quad\left[z, x_{2}\right]+\left[z, x_{2}, x_{2}\right],
$$

and

$$
z+\left[z, x_{1}\right]+\left[z, x_{2}\right]+\left[z, x_{1}, x_{2}\right] \text {. }
$$

It is easily verified that $W_{(2)}$ is an $\operatorname{End}(U)$-submodule. Furthermore, it can be proved that

$$
V_{(2)}=\left\{v \in E+L_{3}:\left[v, x_{i}\right] \in W_{(2)} \text { for } i=1,2\right\} .
$$

Let $M_{(2)}$ be the inverse image of $V_{(2)}$ under $\phi_{3}$ and let $N_{(2)}$ be the inverse image of $W_{(2)}$ under $\phi_{4}$. Then $F_{2} / N_{(2)}$ is a 2-generator relatively free 2-group of nilpotency class 4 with centre $M_{(2)} / N_{(2)}$, which is not fully invariant.

\section{REFERENCES}

[1] R.M. Bryant and L.G. Kovács, 'Lie representations and groups of prime power order', $J$. London Math. Soc. (2) 17 (1978), 415-421. 
[2] B. Huppert and N. Blackburn, Finite groups II (Springer-Verlag, Berlin, Heidelberg, New York, 1982).

[3] W. Magnus, A. Karrass and D. Solitar, Combinatorial group theory (Interscience, New York, 1966).

[4] B.H. Neumann and H. Neumann, 'Zwei Klassen charakteristischer Untergruppen und ihre Faktorgruppen', Math. Nachr. 4 (1951), 106-125.

[5] H. Neumann, Varieties of groups (Springer-Verlag, Berlin, Heidelberg, New York, 1967).

[6] P.M. Neumann, 'A note on formations of finite nilpotent groups', Bull. London Math. Soc. 2 (1970), 91.

[7] M.R. Vaughan-Lee, 'Characteristic subgroups of free groups', Bull. London Math. Soc. 2 (1970), 87-90.

\section{Department of Mathematics \\ University of Manchester Institute of Science and Technology \\ Manchester M60 1QD \\ United Kingdom}

